\title{
A mobile complex to record several secondary cosmic rays components
}

\author{
Evgenya Mikhalko*, Evgeny Maurchev \\ Polar Geophysical Institute, 184209 Apatity, 26A Academgorodok, Russia.
}

\begin{abstract}
The paper presents a research of the different components variations of the secondary cosmic rays (SCR). These are being monitored at the Cosmic Rays Laboratory, PGI, Apatity, using an integrated set based on the SCR basic components detectors. Also, besides stationary equipment, a mobile complex has been developed and made, which consists of a scintillation spectrometer, a charged component detector (CCD) based on the Geiger-Muller counters, and a neutron component detector $(\mathrm{E}<1 \mathrm{MeV})$ based on helium counters SNM-18. The mobile complex was put into operation in the early 2019. It operates in parallel with the basic equipment, recording SCRs. Small dimensions, effective energy consumption and ability to record data onto flash drives allows this complex to be used in SCR-monitoring in remote places, as well as on ships away at sea.
\end{abstract}

\section{Introduction}

When high-energy particles of the primary cosmic radiation pass through the Earth's atmosphere, there appears a cascade of particles various in type and different in energy [1].

The particles flux forms a secondary cosmic radiation (SCR) which is recorded at Polar Geophysical Institute (PGI), Apatity, with an integrated monitoring system developed and made at PGI. The complex consists of detectors recording different SCR components, which allows accumulating an extensive database. Based on the detectors available and on specified tasks, mobile recording devices have been made. Due to the geometric parameters, simplicity of the set and their ability to operate in a self-contained regime, the detectors were integrated into a mobile complex to record several SCR components.

The complex integrates a scintillation spectrometer, a charged component detector (CCD) based on the Geiger-Muller counters, and a neutron component detector $(\mathrm{E}<1$ $\mathrm{MeV}$ ) based on helium counters SNM-18.

\section{A Stationary Complex for Cosmic Rays Recording}

The secondary particles fluxes consisting of the SCR electromagnetic, hadronic and charged components are recorded by detectors of different types: a neutron monitor (NM),

\footnotetext{
*Corresponding author: jane raven@mail.ru
} 
an NM leadless section, a thermal neutron detector, a charged and electromagnetic components detector.

The detectors are integrated into a complex to record SCRs. Each instrument integrated into the complex is assigned to perform its own tasks. For instance, a neutron monitor is integrated into the world neutron monitor network and provides the data into the world database online. However, the information is parallel stored in the common database.

Besides, auxiliary meters (for pressure, precipitation, temperature, and exact time signals source) are common and send signals to a common data bus.

The recording efficiency has been calculated with the software package GEANT-4 for all the detectors, which allowed verifying energy range and recording efficiency. For instance, Figure 1 shows the designed recording efficiency for gamma-radiation quanta for all the scintillation crystals.

It is with this setup that the electromagnetic flux increases in the earth's layer of the atmosphere were for the first time observed during precipitation of various nature (rain, snow, fog) by the Laboratory of PGI [2,3]. To record the electromagnetic SCR-component, scintillation $\mathrm{NaI}(\mathrm{Tl})$-crystal spectrometers of various dimensions are used.

To shield the background radiation from the ground and the surrounding subjects, the detector is covered by lead bricks of 5-cm thick; a window facing upward is left open. It is also necessary to mention that the increases observed are not related to the radioactive pollution of precipitation, and occur only in the electromagnetic component of cosmic rays and are absent in the charged one [3].

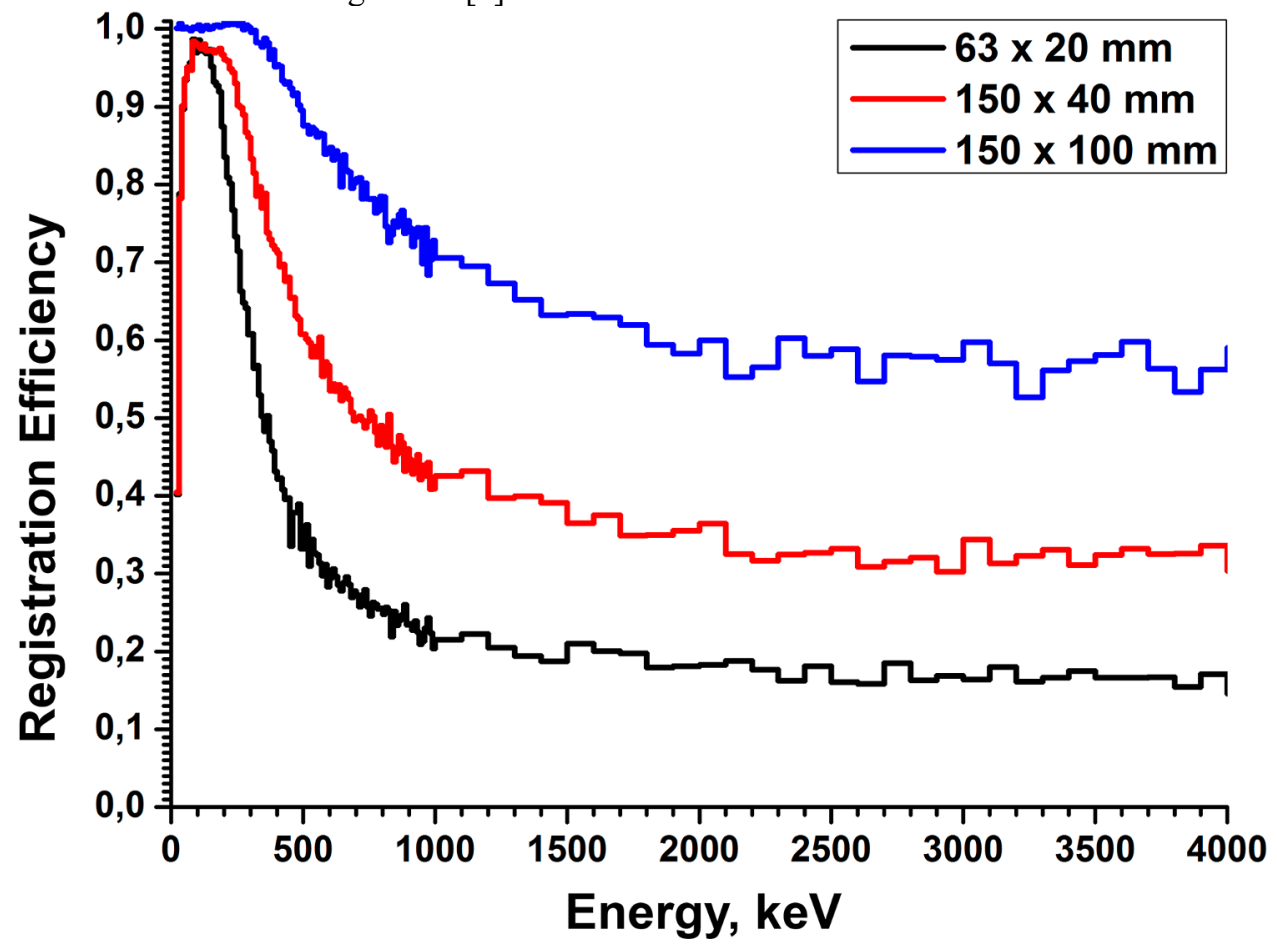

Fig.1. Registration efficiency of the scintillation spectrometer, depending on the NaI(Tl) crystal size. The first number in the crystal designation is its diameter.

To separate the charged component from the electromagnetic one, a charged component detector (CCD) has been developed and installed at PGI. The main detecting elements of the detector are 16 Geiger-Muller (STS-6) counters, arranged in 2 rows, 8 counters in each, separated from each other with a aluminum plate of 4-mm thick. In each layer, the counters 
are parallel connected, according to logic scheme "or"; the rows are connected with each other in logic scheme "and". Signals from the lower row arrive only if signals of the upper and lower rows coincide. The upper row of counters records electrons, muons and positrons of $\mathrm{E}>2 \mathrm{MeV}$; the lower row - electrons, positrons and muons of $\mathrm{E}>\sim 7 \mathrm{MeV}$. As a result, under such connection, CCD has two output channels (a - a channel to count signals arriving from the upper rows of counters, $b-a$ channel of coincidence of pulses from the upper rows), according to energies [5].

Among the basic advantages one can distinguish high performance and ability to operate in a self-contained regime, as well as being of suitable dimensions, which, in turn, are due to a small power source and small counters STS-6.

Like a scintillation spectrometer, it is necessary to place CCD into a lead brick block. It will help to eliminate recording of the background radiation from other subjects and record the charged particles flux only from the upper semi-sphere.

Figure 2 shows the example of the CCD-operation. The charged component variation corresponds to the typical annual variation related to the seasonal changes of the soft muon flux. Figure 2 shows that in both channels, the charged particles flux decreases in the summer period and increases with the winter season coming. A sharp failure in counting in the lower plot coincides with the similar jumps in the thermal neutron's detectors and in the leadless NM section, occurring each year. It seems to show the atmosphere transformation for the warm season.

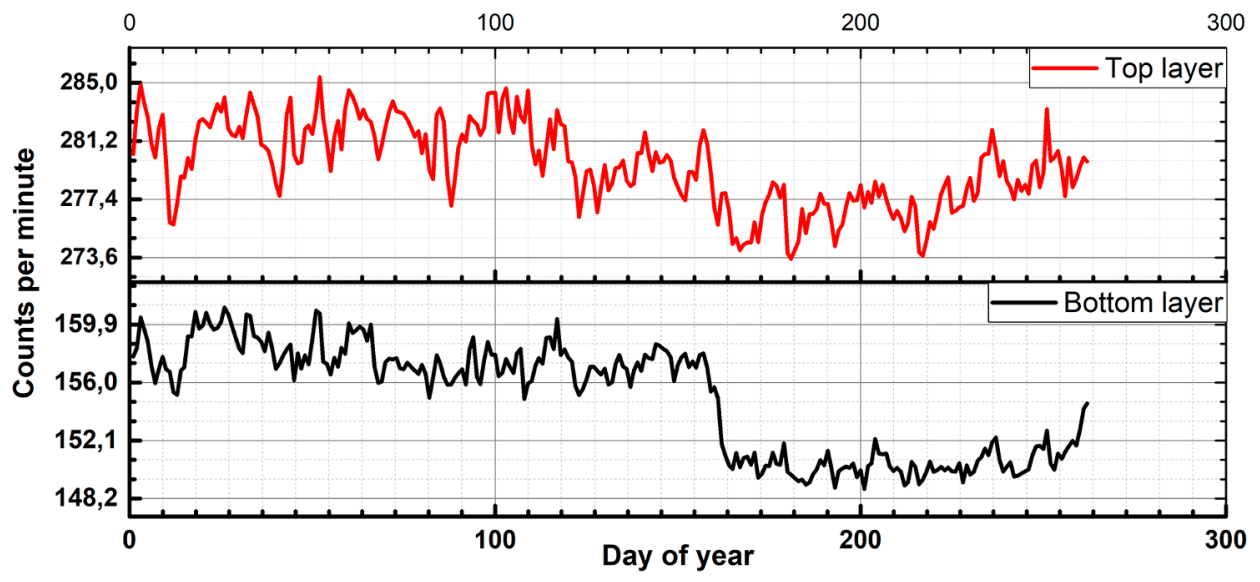

Fig.2. The profile of the charged component counting during several months of the current 2019year a) the $>2 \mathrm{MeV}$ recording channel (the upper row of counts); b) the $>7 \mathrm{MeV}$ recording channel (coincidence between the lower and upper rows counting).

To record the neutron component, there is a complex of equipment at PGI, including a conventional neutron monitor 18-NM-64 (HM), a leadless NM section (LLNMS), and detectors of smaller dimension among which there is a mobile neutron detector.

A standard 18-NM-64 neutron monitor consists of 18 SNM-15 counters covered by lead and polyethylene, records neutrons of $\mathrm{E}>50 \mathrm{MeV}$ in energy [4]. The NM-leadless section is sensitive to neutrons of $(0.1 \div 1) \mathrm{MeV}$ in energy, which has been confirmed by the calculations at the stage of NM-leadless section modeling with software package GEANT4.

The set is equipped with four similar counters SNM-15 covered by polyethylene to moderate neutrons. CR-monitoring with the leadless section has been carried out since 2008 , which significantly extended the energy range of the nucleon component recording.

Note that the standard NM was specially designed not to be sensitive to the local variations of the nucleon flux (atmosphere temperature variations, seasonal changes, 
changes in local environment, etc.). On the contrary, the leadless section's energy threshold is decreased. It is designed to record all the nucleon flux variations. The combination of these two detectors allows the variations to be separated by their origin: local or cosmic.

\section{The Mobile Complex}

The neutron monitor (NM) dimensions and weight are great ( 9 meters long and 48 tons). As for the leadless section, its weight is much less but it is 2 meters long. These detectors are stationary. Due to their great area, these detectors are also characterized by high rate of counting, which gives a possibility to use small time resolution -1 minute.

The mobile complex has been developed because of great dimension and non-mobility of basic detectors. This complex consists of three basic SCR- components. A small NaI(Tl) crystal is used as an electromagnetic component detector. Figure 1 shows that small detectors $(\varnothing 63 \times 20 \mathrm{~mm})$ are highly effective in the recording range of $20-400 \mathrm{KeV}$.

A mobile charged-component detector is a unit composed of eight Geiger-Muller counters, with a modernized electronic part and a high voltage source of a compact size. The CCD of the given configuration records electrons and low-energy muons of $>2 \mathrm{MeV}$ in energy, which is sufficient to carry out observations over the annual seasonal variations.

Based on the leadless section, the mobile neutron detector (ND) has been developed. Eight helium counters SNM-18 operate as detecting elements. The counters are placed in a polyethylene box, with walls of $2.5 \mathrm{~cm}$ thick. The SNM-18, like SNM-15, is sensitive only to thermal neutrons. If, however, the detectors are covered by polyethylene to moderate neutrons, it will be possible to extend the energy range to $1 \mathrm{MeV}$. The lead shield absence makes this detector sensitive to different local variations of the neutron background, including those related to radon emissions.

The data acquisition system has been also improved. A modern microcontroller is used instead of the computer to record information to a solid-state drive (a flash drive). It allows the complex to be used as an independent instrument. Small dimensions and weight (less than $20 \mathrm{~kg}$ ), low power consumption (less than 20 watt) make the complex mobile, simple to install and operate.

Figure 3 shows the neutron detector count profile for a month and a half, since the moment the mobile complex was put into operation.

To develop of the complex, use was made of up-to-date and high-speed components. It allowed the arrival time of every pulse to be recorded with high accuracy $(2 \mu \mathrm{s})$, in addition to a conventional way of data acquisition, which fixes the detector counting rate. It makes it possible to identify the effects arising out of CR passing through the atmosphere Accurate recording of each particle arrival allows determining the temporal intervals between particles arrival to the detectors, and building the distribution of the intervals according to their duration. This distribution is shown in Fig.4. The distribution was being built as follows. All the number of the intervals accumulated in the course of the set operation (250000 intervals) was distributed as follows: the number of 0-50 ms intervals was calculated and the value obtained was recorded into the first bin of distribution. The number of 50-100 ms intervals was recorded into the second bin, etc. In this distribution, the size of the bin is $50 \mathrm{~ms}$. The distribution is shown Fig.4, bottom. In the second case, the size of the bin was specified being equal to $2 \mu \mathrm{s}$. The distribution was built in a similar way: the number of $0-2 \mu$ s intervals was recorded into the first bin, the number of 2-4 $\mu$ s intervals was recorded into the next bin, etc. 


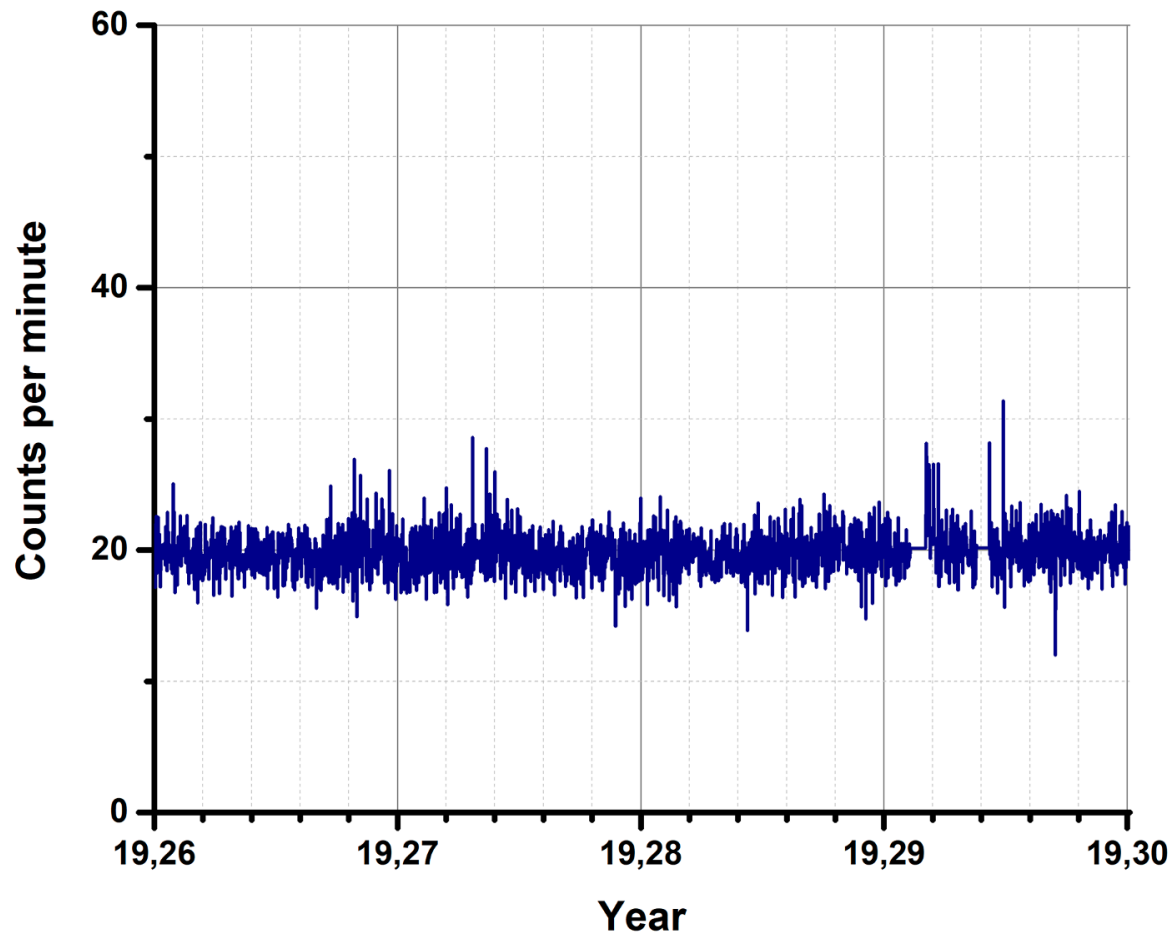

Fig.3. The neutron component count profile by the mobile complex data in 2019 .

The distribution is shown in Fig.4, top. The form of distribution in Fig.4 (bottom) is an exponent (a line in a semi-log scale). This is a reliable indication of that the particles arrive to the detector randomly, and the particles arrival is defined by the Poisson law [5]. The exception was the first bin, which contained the exceeded number of short intervals. To get a detail distribution in short intervals, the distribution was built, in which the size of bin was specified as being equal to $2 \mu \mathrm{s}$. The reason was obvious: the number of intervals accumulated (250000) was not enough to fill all the cells and to build this distribution with high accuracy.

It is vividly seen, however, that there are too many very short intervals (less than 100 $\mu \mathrm{s})$ - by a factor of $10^{2}$ greater than it is necessary under random arrival of particles. This abnormal phenomenon indicates that the arrival of two or three particles within the interval of $100 \mu \mathrm{s}$ was not an occasional one. An isolated data analysis showed that there were 1528 cases of a couples recording of neutrons, 3169 cases of triple recording, and 161 cases of even quadruple recording. It means that the overwhelming part of these events occurred during high density of neutrons in the environment. This is possible when there appear extended atmospheric showers in the atmosphere (EAS) [4].

One energy particle of the cosmic rays produces a shower of particles of different type: electrons, muons, nucleons, $\pi$-mesons in the Earth's atmosphere. The total number of particles id as great as $10^{6}$. This flux of particles is propagated throughout the atmosphere and, subsiding, achieves the earth's surface to be recorded by various instruments.

Thus, high speed software in combination with the corresponding electronic inroute allows the arrival of separate particles of high energies to be recorded on the detector. This peculiarity of the complex is of interest in that the detectors (WAS) are stationary complexes. 


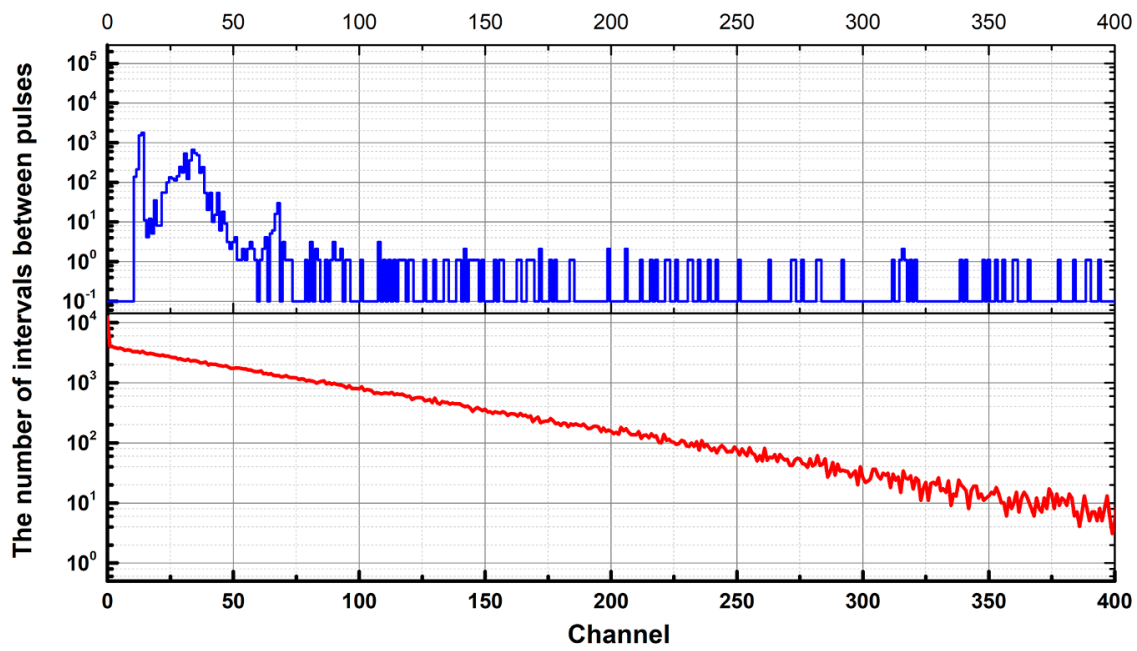

Fig.4. The temporal intervals distribution between the pulses. The upper panel is consistent with the distribution with the temporal bin of $2 \mu \mathrm{s}$, the lower panel is consistent with the distribution with the temporal bin of $50 \mathrm{~ms}$. For the upper plot, in order to get the value of the interval in $\mu \mathrm{s}$, the number of the channel (on X axis) should be multiplied by 2. For instance, channel 50 corresponds to the temporal interval 100-102 $\mu \mathrm{s}$. For the bottom plot, in order to get the value of the interval in $\mathrm{ms}$, the number of channels should be multiplied by 50 .

\section{Conclusion}

A mobile complex developed at PGI is presented to be used in recording the different components of secondary cosmic rays. It is designed to make observations over the variations of cosmic rays in the course of expeditions, as well as to be used in the localities difficult to reach.

The significant peculiarity of the complex is its universality: the neutron, charged particles and electromagnetic components detectors are integrated into a system. The detector construction makes it sensitive to neurons of moderate energies (to $1 \mathrm{MeV}$ ) and is able to record the variations as of both cosmic nature and local ones related to the peculiarities of the topography.

The study has been supported by the RSF grant 18-77-10018

\section{References}

1. S. Hayakawa, Cosmic Ray Physics. Nuclear and Astrophysical aspects (WileyInterscience, New York, 1969)

2. A.V. Germanenko, Yu.V. Balabin, E.V. Vashenyuk, B.B. Gvozdevsky, L.I. Schur, Astrophys. Space Sci. Trans., 7, 471 (2011)

3. J.V. Balabin, A.V. Germanenko, B.B. Gvozdevsky, E.V. Vashenjuk, Geomagnetism and aeronomy, 54, 376 (2014)

4. Dorman L.I. Experimental and theoretical fundamental astrophysics of cosmic rays (NAUKA, Moscow, 1975) (in Russian)

5. V.I. Goldansii, A.V. Kucenko, M.I. Podgoretskii, Counting statistics for registration of nuclear particles (FIZMATGIZ, Moscow, 1959) (in Russian) 